\title{
O conhecimento do professor de educação física sobre aprendizagem motora
}

\section{The knowledge of the physical education teacher about motor learning}

\section{El conocimiento del profesor de educación física sobre el aprendizaje motor}

iD Nathálya Gardênia de Holanda Marinho Nogueira Universidade Federal de Minas Gerais - UFMG, Belo Horizonte, MG, Brasil marinhohnathy@gmail.com

iD Diego Augusto Paulon Universidade Federal de Minas Gerais - UFMG, Belo Horizonte, MG, Brasil diegoaugustopaulon@hotmail.com

iD Bárbara de Paula Ferreira Universidade Federal de Minas Gerais - UFMG, Belo Horizonte, MG, Brasil barbaradepaulaf@gmail.com

(D) Lidiane Aparecida Fernandes Universidade Federal de Minas Gerais, Belo Horizonte, MG, Brasil lidianefernandes12@yahoo.com.br

iD Guilherme Menezes Lage Universidade Federal de Minas Gerais, Belo Horizonte, MG, Brasil menezeslage@gmail.com

Resumo: O objetivo deste estudo foi investigar o nível de conhecimento de graduados e graduandos em Educação Física sobre Aprendizagem Motora. Para isso, foi elaborado um questionário com 20 questões, sendo a primeira parte relacionada à identificação dos participantes e a segunda relacionada à Aprendizagem Motora. A análise foi feita com base na frequência de respostas dos participantes. A maioria dos participantes afirmou que sabiam o que era Aprendizagem Motora. No en- 
tanto, exibiram dificuldades em relação a algumas informações sobre a parte teórica. Além disso, os participantes apresentaram dificuldade em responder questões relacionadas aos fatores que influenciam a aprendizagem motora. Apesar do conhecimento sobre a temática contribuir efetivamente na forma pela qual o professor de educação física planeja e conduz as suas aulas, os participantes apresentaram impasses em responder às questões.

Palavras-chave: Comportamento Motor. Educação Física. Habilidades Motoras. Atuação Profissional.

Abstract: The study aimed to investigate the level of knowledge of undergraduate and graduate students in physical education on Motor Learning. For this, a questionnaire with 20 questions was elaborated, the first part related to the identification of the participants and the second related to Motor Learning. The analysis was made based on the frequency of responses from the participants. Most of the participants stated that they knew what Motor Learning was. However, they showed difficulties about some information about the theoretical part. Also, the participants had difficulty in answering questions related to the factors that influence motor learning. Despite the knowledge about the theme effectively contributing to the way the physical education teacher plans and conducts his classes, the participants presented impasses in answering the questions.

Keywords: Motor Behavior. Physical Education. Motor Skills. Professional Performance.

Resumen: El objetivo del estudio fue investigar el nivel de conocimiento de estudiantes de pregrado y posgrado en educación física sobre el aprendizaje motor. Para esto, se elaboró un cuestionario con 20 preguntas, la primera parte relacionada con la identificación de los participantes y la segunda relacionada con el aprendizaje motor. El análisis se realizó en función de la frecuencia de las respuestas de los participantes. La mayoría de los participantes declararon que sabían lo que era el aprendizaje motor. Sin embargo, mostraron dificultades 
en relación con alguna información sobre la parte teórica. Además, los participantes tuvieron dificultades para responder preguntas relacionadas con los factores que influyen en el aprendizaje motor. A pesar de que el conocimiento sobre el tema contribuyó efectivamente a la forma en que el maestro de educación física planifica y dirige sus clases, los participantes presentaron dificultades para responder las preguntas.

Palabras clave: Comportamiento Motor. Educación Física. Habilidades Motoras. Actuación Profesional.

Submetido em: 16-07-2020

Aceito em: 05-08-2020 
O conhecimento do professor de educação física sobre aprendizagem motora Nathálya Gardênia de Holanda Marinho Nogueira • Diego Augusto Paulon • Bárbara de Paula Ferreira • Lidiane Aparecida Fernandes • Guilherme Menezes Lage

\section{Introdução}

A alteração na capacidade do aprendiz em desempenhar uma habilidade motora, inferida pela melhoria relativamente permanente no desempenho motor, a partir de prática ou experiência, é definida como aprendizagem motora (MAGILL, 2000). Segundo Araújo et al. (2012), as habilidades motoras abrangem um amplo grupo de movimentos direcionados a metas, destacando-se as habilidades posturais, para manter o corpo em orientações específicas em relação ao ambiente; as habilidades locomotoras, relacionadas ao deslocamento do corpo e as habilidades manipulativas para explorar e interagir com os objetos no ambiente. Sabe-se que o ser humano executa essas habilidades em variados contextos, como no esporte, no trabalho, na escola e em outras situações do cotidiano (ARAÚjO et al., 2012).

A Aprendizagem Motora como área de estudo investiga os fatores que influenciam o processo de aprendizagem das habilidades motoras (UGRINOWITSCH; BENDA, 2011). Dentre esses fatores, destaca-se a forma pela qual a prática é estruturada, que pode ser de forma constante ou variada. A prática constante envolve a prática de apenas uma habilidade (por exemplo, AAAAAAAAA), e a prática variada envolve a prática de duas ou mais habilidades ou variações de uma mesma habilidade (LELIS-TORRES et al., 2017). Existem algumas formas de estruturar a prática variada, dentre elas duas têm se destacado nos últimos estudos sobre interferência contextual: a prática em blocos e a prática aleatória, talvez por serem os dois extremos utilizados para se estruturar a prática variada (LAGE et al., 2011). A prática variada em blocos consiste na prática de blocos de tentativas de cada uma das habilidades a serem aprendidas, dessa forma o aprendiz executa todas as tentativas de uma habilidade antes de iniciar um novo bloco de tentativas de outra habilidade (por exemplo, AAABBBCCC). A prática variada aleatória consiste em praticar as habilidades em uma ordem não sistemática para o aprendiz, de modo que haja 
O conhecimento do professor de educação física sobre aprendizagem motora

Nathálya Gardênia de Holanda Marinho Nogueira • Diego Augusto Paulon • Bárbara de Paula

Ferreira • Lidiane Aparecida Fernandes • Guilherme Menezes Lage

uma constante mudança de habilidade a cada nova tentativa (por exemplo, ABCBCACAB) (LAGE et al., 2011). Apesar da prática em blocos conduzir um desempenho superior durante a fase de aquisição, quando o desempenho dos grupos é comparado nos testes de retenção e transferência, a prática aleatória conduz a um desempenho superior em relação à prática em blocos (GONÇALVES et al., 2007; LIN et al., 2013).

Um segundo fator que influencia a aprendizagem motora é a forma pela qual a informação é transmitida ao aprendiz, que pode ser a partir de instrução verbal e demonstração. Essas formas de informações prévias à realização da ação são os dois fatores mais frequentemente utilizados como instrumentos de ensino (PÚBLIO; TANI; MANUEL, 1995). As informações sobre a meta, a especificação e o modo de execução da tarefa, quando verbalizadas ou apresentadas de forma escrita, são definidas como instrução verbal. Através da transmissão dessas informações, o professor procura esclarecer o padrão de movimento que o aprendiz deve alcançar ao final da prática (TANI, 1989). A forma de fornecer informação relacionada ao padrão espaço-temporal de uma ação a ser realizada para alcançar a meta é definida como demonstração. Especificamente, a demonstração é entendida como uma informação sobre "como fazer" (SANTOS-NAVES et al., 2014), em que o aprendiz observa um modelo. De acordo com Tonello e Pellegrini (1998), durante a demonstração, os observadores tendem a codificar, classificar e reorganizar os elementos da tarefa em esquemas familiares para recordar mais facilmente e, assim, a explicação por modelo pode ser frequentemente utilizada como estratégia de aprendizagem de novas habilidades motoras. Ainda em relação à demonstração, é interessante que o professor utilize recursos para que os alunos tenham ideia da habilidade a ser executada, como: o próprio professor ou um aluno com conhecimento da habilidade motora ser o modelo ou até mesmo a utilização de vídeos. Dessa forma, é fundamental que o professor saiba transmitir informações rápidas e precisas, fazendo uso de instrução verbal e demonstração para que os alunos não sejam confundidos antes de realizarem a tarefa. 
O conhecimento do professor de educação física sobre aprendizagem motora Nathálya Gardênia de Holanda Marinho Nogueira • Diego Augusto Paulon • Bárbara de Paula Ferreira • Lidiane Aparecida Fernandes • Guilherme Menezes Lage

Um terceiro fator que influencia na aprendizagem de habilidades motoras é o feedback, que são informações sobre como está sendo executado o movimento ou informações após a execução do movimento, que permitem ao aprendiz avaliar se o movimento executado alcançou ou não a meta (TANI; JUNIOR; GOMES, 2005). De acordo com Corrêa et al. (2005), o feedback pode ser intrínseco, que se refere à informação sensorial que surge naturalmente da produção do movimento, e o feedback extrínseco, que se refere a uma informação sobre o movimento, que é fornecida como um complemento ao feedback intrínseco. Existem dois tipos de feedback extrínseco, o Conhecimento de Performance (CP) e o Conhecimento de Resultados (CR) (CORRÊA et al., 2005). O CP fornece informações sobre como o movimento está sendo e/ou foi executado e o CR fornece informações sobre o resultado do movimento em relação à meta a ser alcançada (TANI, 1989). Dessa forma, o conhecimento sobre a área da Aprendizagem Motora, principalmente em relação aos fatores que podem ser manipulados pelo professor no processo de ensino-aprendizagem, pode contribuir efetivamente na forma pela qual o professor de educação física planeja e conduz suas aulas e possíveis intervenções. Esse conhecimento é fundamental não somente para profissionais já atuantes mas também para futuros profissionais da área. Assim, o objetivo do presente estudo foi investigar o nível de conhecimento dos graduados e graduandos em Educação Física sobre a área da Aprendizagem Motora.

\section{Métodos}

\section{Amostra}

Participaram deste estudo 218 graduados e graduandos em Educação Física, de ambos os sexos. Foram incluídos no estudo graduados que atuassem na área e formados nas modalidades de bacharelado, licenciatura ou em ambas, e graduandos de qualquer período nas modalidades de bacharelado, licenciatura ou em 
O conhecimento do professor de educação física sobre aprendizagem motora Nathálya Gardênia de Holanda Marinho Nogueira • Diego Augusto Paulon • Bárbara de Paula Ferreira • Lidiane Aparecida Fernandes • Guilherme Menezes Lage

ambas. Inicialmente, a amostra consistiu em 218 participantes. No entanto, os resultados de 77 participantes, que declararam não saber o que é Aprendizagem Motora ou desistiram de continuar respondendo ao questionário, não foram analisados na segunda parte da pesquisa. O comitê de ética da universidade local aprovou todos os procedimentos ( $\mathrm{n}^{\circ}$ de protocolo: 52865815.9.1001.5153), em conformidade com os padrões estabelecidos pela Declaração de Helsinque (versão 2014).

\section{Instrumento e tarefa}

Foi elaborado um questionário com a finalidade de analisar o conhecimento de graduados e graduandos em Educação Física, que abordou temas relacionados à Aprendizagem Motora. $O$ questionário foi composto por vinte questões, sendo que as seis primeiras foram destinadas à identificação profissional do participante e as demais questões à análise do conhecimento específico sobre Aprendizagem Motora. A seção 1 foi composta pelas questões 7-10, que se referiam ao conhecimento teórico geral sobre a área, a seção 2 foi composta pelas questões 11-13, abordando as diferentes classificações das habilidades motoras e a seção 3 foi composta pelas questões 14-19, envolvendo os fatores que influenciam a aprendizagem motora. A questão 20 buscou verificar a opinião pessoal dos participantes sobre a importância da Aprendizagem Motora na prática profissional.

\section{Procedimentos}

Os graduados e graduandos em Educação Física foram convidados a participar deste estudo a partir de redes sociais e e-mails. Os participantes recebiam uma mensagem contendo informações a respeito dos objetivos da pesquisa juntamente com um link que os redirecionava para a página do questionário. Para a elabora- 
O conhecimento do professor de educação física sobre aprendizagem motora

Nathálya Gardênia de Holanda Marinho Nogueira • Diego Augusto Paulon • Bárbara de Paula

Ferreira • Lidiane Aparecida Fernandes • Guilherme Menezes Lage

ção do questionário foi utilizado um site específico para essa finalidade (SurveyMonkey) e, a partir disso, foi gerado um link para o questionário. Foi feita uma análise descritiva com a frequência de respostas dos participantes. Essa análise foi apresentada através da frequência relativa.

\section{Resultados}

Os resultados foram obtidos a partir das respostas do questionário, sendo que a primeira parte do questionário consistiu em questões relacionadas à identificação de graduados e graduandos em Educação Física (218 participantes) e a segunda parte consistiu em questões relacionadas à Aprendizagem Motora (141 participantes). A primeira parte do questionário estava relacionada à identificação dos participantes (questões 1-6). Assim, 59,17\% eram graduados em Educação Física e os outros 40,83\% eram graduandos. Dos graduados, 40,28\% eram formados nas duas modalidades, Bacharelado e Licenciatura, 31,25\% apenas em Bacharelado e $28,47 \%$ apenas em Licenciatura, sendo que a maior parte dos graduados, 52,24\%, era formada há menos de cinco anos. Além disso, em relação aos graduandos, $46,43 \%$ dos participantes estavam no Bacharelado, $35,71 \%$ na Licenciatura e os outros $17,86 \%$ em ambas as modalidades. Finalizando a primeira parte do questionário, do total de respostas obtidas, 91,28\% participantes responderam que estudaram ou estudam algum conteúdo referente às disciplinas de Aprendizagem Motora ou Comportamento Motor. Dos participantes que responderam ao questionário, 96,33\% afirmaram que sabiam o que é Aprendizagem Motora e a partir dessas respostas foi dada a continuidade à segunda parte do questionário. Para aqueles que responderam que não sabiam, 3,67\%, o questionário foi automaticamente finalizado.

A segunda parte do questionário estava relacionada ao conhecimento sobre Aprendizagem Motora e foi particionada em três seções (questões 7-19) e a questão 20. A média de respostas corretas 
O conhecimento do professor de educação física sobre aprendizagem motora Nathálya Gardênia de Holanda Marinho Nogueira • Diego Augusto Paulon • Bárbara de Paula Ferreira • Lidiane Aparecida Fernandes • Guilherme Menezes Lage

na seção 1 está representada na Figura 1, essa seção foi composta pelas questões 7-10 que se referiam ao conhecimento teórico geral sobre a área. A questão 7 continha nas alternativas os conceitos de Desenvolvimento Motor, Aprendizagem Motora e Controle Motor. Em relação ao conceito de Aprendizagem Motora, 42,55\% dos participantes responderam que todas as alternativas estavam corretas, 27,66\% responderam corretamente o conceito de Aprendizagem Motora, $19,86 \%$ responderam o de Desenvolvimento Motor, 5,67\% o de Controle Motor e 4,26\% responderam que nenhuma das alternativas conceituava Aprendizagem Motora.

$\mathrm{Na}$ questão 8 havia alternativas com teorias e modelos do Comportamento Motor, sendo que duas delas eram diretamente teorias da Aprendizagem Motora. A intenção desta questão era investigar se os participantes possuíam conhecimento de uma ou mais teorias da Aprendizagem Motora. No entanto, as duas alternativas mais escolhidas foram a Teoria de Esquema de Schmidt e o Modelo da Ampulheta de Gallahue e Ozmun, com 33,33\% e $34,75 \%$, respectivamente. A Teoria da Aprendizagem Social de Albert Bandura obteve $8,51 \%$ de escolhas e a Teoria do Circuito Fechado de Adams, 18,44\%. Nas alternativas de respostas como "todas as opções anteriores" e "nenhuma das opções anteriores", a primeira alcançou $23,40 \%$ e a segunda $4,96 \%$.

A questão 9 foi sobre as fases da aprendizagem motora, em que havia um enunciado sobre uma das fases e o participante deveria assinalar se a opção era relacionada à fase cognitiva, associativa ou autônoma. Quando o enunciado citava a fase cognitiva, $76,60 \%$ das respostas foram corretas, $17,73 \%$ escolheram a opção que se referia à fase associativa e 5,67\% à fase autônoma. No enunciado sobre a fase autônoma, 55,32\% marcaram corretamente, $35,46 \%$ escolheram a opção que se referia à fase associativa e $9,22 \%$ à fase cognitiva. Por fim, quando a informação trazida era a respeito da fase associativa, $48,23 \%$ assinalaram a opção condizente ao enunciado, 39,01\% como fase autônoma e 12,77\% como fase cognitiva. 
O conhecimento do professor de educação física sobre aprendizagem motora Nathálya Gardênia de Holanda Marinho Nogueira • Diego Augusto Paulon • Bárbara de Paula Ferreira • Lidiane Aparecida Fernandes • Guilherme Menezes Lage

A questão 10 estava relacionada aos fatores que o professor pode manipular na prática para favorecer a aprendizagem motora dos seus alunos. De acordo com as respostas obtidas, 37,59\% afirmaram que "feedback extrínseco, observação, organização da prática e instrução verbal" eram esses fatores, 17,73\% para "propriocepção, demonstração, feedback e organização da prática", 19,15\% para "prática mental, feedback extrínseco, demonstração e organização da prática" e 25,53\% para "instrução verbal, feedback extrínseco, tato e organização da prática".

A média de respostas corretas na seção 2 está representada na Figura 1. Essa seção foi composta pelas questões 11-13, abordando as diferentes classificações das habilidades motoras. Nas questões 11-13, os participantes tinham que relacionar a primeira coluna em relação à classificação das habilidades motoras com as habilidades de nadar 25 metros em uma raia, correr nas ruas, executar o tiro livre do basquetebol, correr e pular uma série de obstáculos, rebater uma bola de beisebol e posicionar uma peça no xadrez. A questão 11 foi sobre a classificação de habilidade motora grossa ou fina, a questão 12 foi sobre a classificação das habilidades motoras como discreta, seriada ou contínua e a questão 13 foi sobre a classificação como habilidade motora aberta ou fechada. Na questão $11,86,52 \%$ dos participantes consideraram "nadar 25 metros em uma raia" como habilidade motora grossa e $13,48 \%$ como habilidade motora fina. Quando a questão foi sobre "correr nas ruas", 97,86\% dos participantes responderam que era uma habilidade motora grossa e 2,14\% como habilidade motora fina. Para o "executar o tiro livre do basquetebol", 44,29\% dos participantes a consideraram uma habilidade motora grossa e para $55,71 \%$ uma habilidade motora fina. A opção "correr e pular uma série de obstáculos" foi considerada para $80,85 \%$ dos participantes uma habilidade motora grossa e para $19,15 \%$ uma habilidade motora fina. Respectivamente, $53,19 \%$ e $46,81 \%$ dos participantes consideraram habilidade motora grossa e fina para "rebater uma bola de beisebol". Por fim, na habilidade motora "posicionar uma peça no xadrez", 8,51\% consideraram uma habilidade motora grossa e $91,49 \%$ uma habilidade motora fina. 
O conhecimento do professor de educação física sobre aprendizagem motora Nathálya Gardênia de Holanda Marinho Nogueira • Diego Augusto Paulon • Bárbara de Paula Ferreira • Lidiane Aparecida Fernandes • Guilherme Menezes Lage

Para a questão 12, em relação ao "nadar 25 metros em uma raia", $75,18 \%$ dos participantes responderam que era uma habilidade motora contínua, $17,73 \%$ seriada e 7,09\% discreta. Para o "correr nas ruas", 80,71\% dos participantes responderam que era uma habilidade motora contínua, $10,00 \%$ e 9,29\% como habilidade motora seriada e discreta, respectivamente. Para o "executar o tiro livre do basquetebol", 8,63\% dos participantes responderam que era uma habilidade motora contínua, 40,29\% seriada e 51,08\% discreta. Em relação ao "correr e pular uma série de obstáculos", $17,27 \%$ dos participantes responderam que era uma habilidade motora contínua, 79,14\% seriada e 3,60\% discreta. Para o "rebater uma bola de beisebol", 14,49\% dos participantes responderam que era uma habilidade motora contínua, 25,36\% seriada e 60,14\% discreta. Para o "posicionar uma peça no xadrez", 7,14\% dos participantes responderam uma habilidade motora contínua, 6,43\% seriada e $86,43 \%$ discreta.

Na questão 13, em relação ao "nadar 25 metros em uma raia", $63,12 \%$ e $36,88 \%$ dos participantes a classificaram como habilidade motora fechada e aberta, respectivamente. Para o "correr nas ruas", $75,71 \%$ dos participantes responderam habilidade motora aberta e $24,29 \%$ fechada. Em relação ao "executar o tiro livre do basquetebol", 25,53\% dos participantes responderam habilidade motora aberta e 74,47\% fechada. Para o "correr e pular uma série de obstáculos", 55,32\% dos participantes responderam habilidade motora aberta e 44,68\% fechada. Para o "rebater uma bola de beisebol", 57,45\% dos participantes responderam habilidade motora aberta e $42,55 \%$ fechada e para o "posicionar uma peça no xadrez", 22,14\% dos participantes consideraram como habilidade motora aberta e $77,86 \%$ como fechada.

A média de respostas corretas na seção 3 está representada na Figura 1. Essa seção foi composta pelas questões 14-19, envolvendo os fatores que influenciam a aprendizagem motora. A questão 14 foi relacionada ao feedback, mais especificamente sobre como informar ao aluno sobre o padrão de movimento já executado, 9,22\% dos participantes responderam "por meio de CR"; $17,73 \%$ 
O conhecimento do professor de educação física sobre aprendizagem motora Nathálya Gardênia de Holanda Marinho Nogueira • Diego Augusto Paulon • Bárbara de Paula Ferreira • Lidiane Aparecida Fernandes • Guilherme Menezes Lage

"por meio de CP"; 23,40\%, "fornecer demonstração" e 49,65\% dos participantes responderam que todas as alternativas eram as meIhores formas de informar um aluno sobre o seu padrão de movimento em uma aula de educação física. Em relação à questão 15, os participantes deveriam responder sobre a estruturação da prática, mais especificamente como se estruturar a prática de uma ou mais habilidades motoras: 9,22\% dos participantes responderam que "por meio de prática mental"; 41,84\% "por meio da prática variada aleatória"; 49,65\% "por meio da prática constante" e 4,26\% "por meio da prática observacional".

A questão 16 foi referente à demonstração e a influência exercida por ela na aprendizagem motora, 34,04\% dos participantes responderam que a demonstração "propicia ao aluno a possibilidade de corrigir a habilidade a partir de um modelo", 1,42\% que é "um fator de motivação para o aluno diante de uma nova habilidade", 63,12\% que "permite a formulação de uma representação mental da ação a ser realizada" e 1,42\% não consideraram que a demonstração influencia na aprendizagem. Em relação à questão 17, os participantes deveriam responder se a prática mental era superior a qual ou quais condições de prática, 83,69\% dos participantes responderam que a prática mental era superior a "ausência de prática", enquanto $7,80 \%$ responderam que era superior a "prática constante", 6,38\% a "prática em blocos" e 2,13\% a "prática aleatória".

A questão 18 foi referente à temática da prática pelo todo e em partes, 54,61\% dos participantes responderam que "para tarefas com alta complexidade e baixa organização seria indicada a prática em partes", 15,60\% que "para tarefas com alta complexidade e alta organização seria indicada a prática pelo todo", 7,80\% que "para tarefas com alta organização e baixa complexidade seria indicada a prática mista" e 21,99\% responderam que nenhuma das opções anteriores estava correta. Em relação à questão 19, os participantes deveriam responder sobre instrução verbal e a meIhor forma de fornecê-la, $60,28 \%$ dos participantes responderam "utilizar frases curtas e concisas a respeito da meta da habilidade, 
O conhecimento do professor de educação física sobre aprendizagem motora

Nathálya Gardênia de Holanda Marinho Nogueira • Diego Augusto Paulon • Bárbara de Paula

Ferreira • Lidiane Aparecida Fernandes • Guilherme Menezes Lage

ou seja, usar pistas verbais", 36,17\% que era "fornecer instrução verbal antes, durante e após a execução da habilidade", 2,84\% que era "fornecer várias informações a respeito do erro do aprendiz" e $0,71 \%$ que era "fornecer informações demasiadas para o aluno durante a prática da habilidade". As médias globais de respostas corretas e incorretas das seções 1-3 estão representadas na Figura 2.

Figura 1 - Média de respostas corretas nas diferentes seções. Seção 1 composta pelas questões 7-10; Seção 2 - composta pelas questões 11-13; Seção 3 - composta pelas questões 14-19.

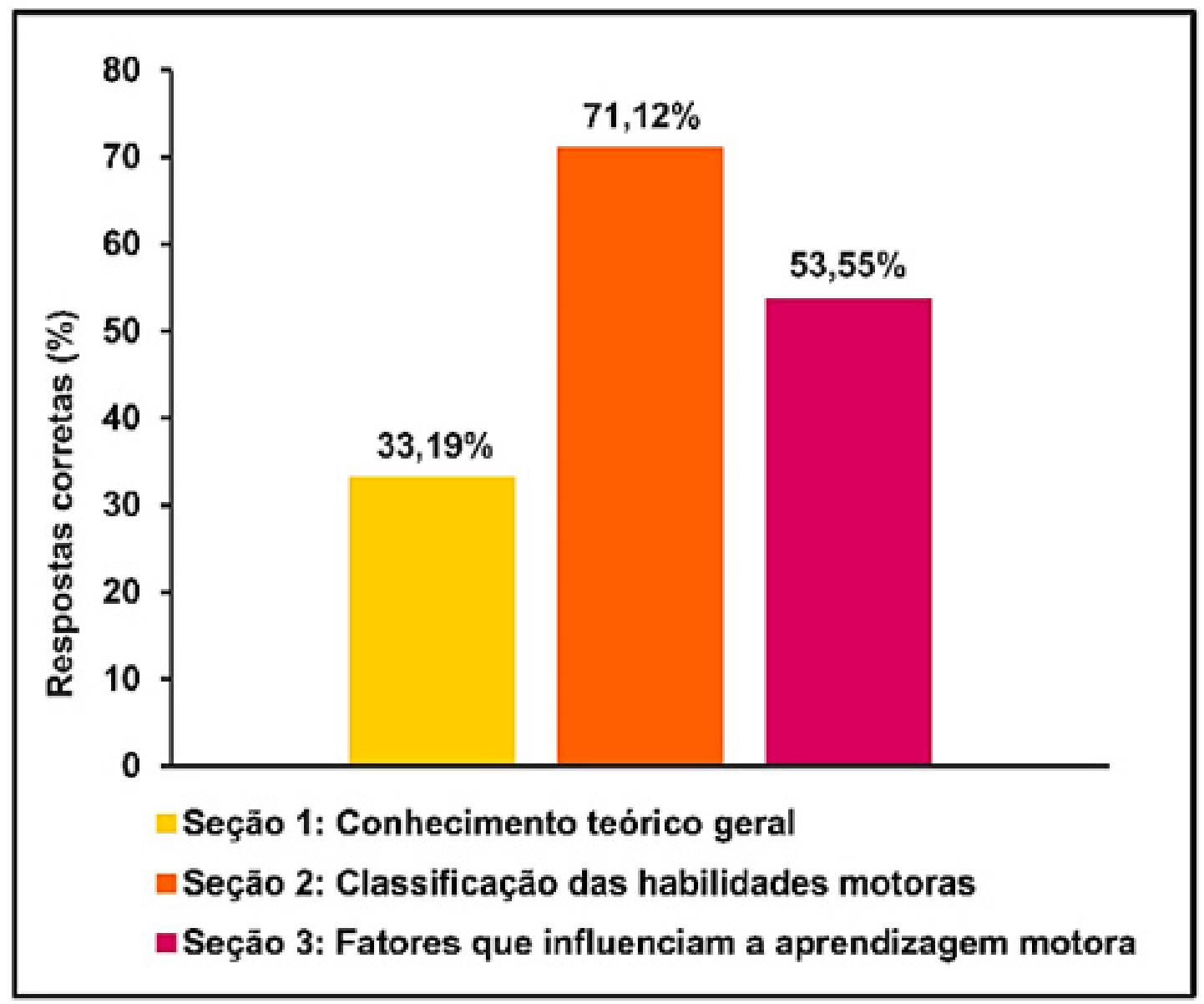

Fonte: autores. 
O conhecimento do professor de educação física sobre aprendizagem motora Nathálya Gardênia de Holanda Marinho Nogueira • Diego Augusto Paulon • Bárbara de Paula Ferreira • Lidiane Aparecida Fernandes • Guilherme Menezes Lage

Figura 2 - Médias globais de respostas corretas e incorretas das seções 1-3.

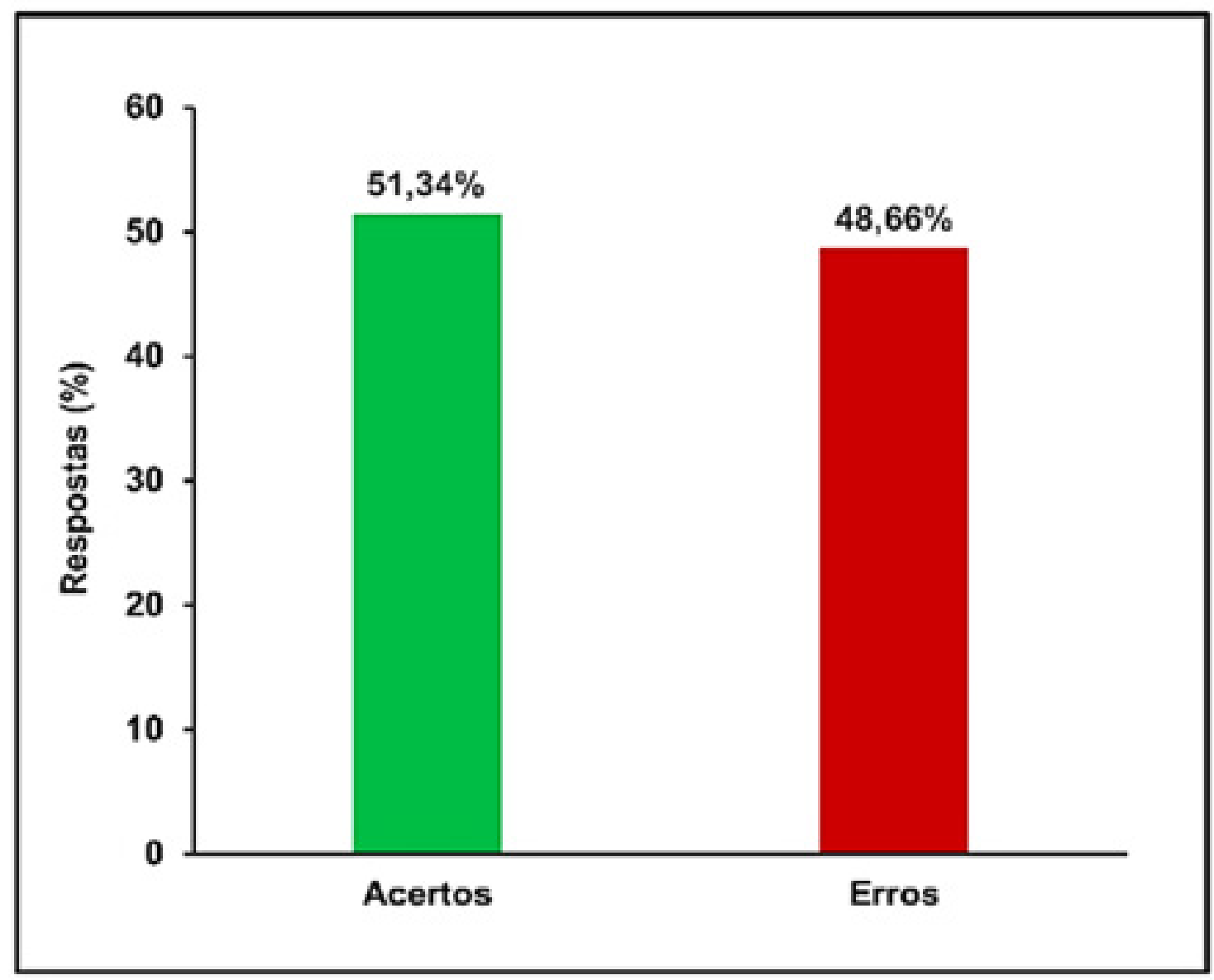

Fonte: autores.

A última pergunta do questionário foi se o participante achava que o conhecimento sobre Aprendizagem Motora era importante para a prática do professor de educação física ou não, 86,52\% dos participantes responderam de muita importância, 12,06\% que era importante e 1,42\% pouca importância.

\section{Discussão}

O objetivo do presente estudo foi investigar o nível de conhecimento dos graduados e graduandos em Educação Física sobre Aprendizagem Motora. A maioria dos participantes afirmou que 
O conhecimento do professor de educação física sobre aprendizagem motora Nathálya Gardênia de Holanda Marinho Nogueira • Diego Augusto Paulon • Bárbara de Paula Ferreira • Lidiane Aparecida Fernandes • Guilherme Menezes Lage

sabia o que era Aprendizagem Motora. Assim, foram analisadas as respostas dos participantes que fizeram essa afirmação. Em relação às questões que os participantes tiveram facilidade em responder, pode-se iniciar pela questão 9, que foi fundamentada segundo o modelo de três estágios de Fitts e Posner (1967): cognitivo, associativo e autônomo. Nota-se, pelos resultados, que os participantes, de maneira geral, responderam corretamente sobre os estágios de aprendizagem. Vale ressaltar que o professor de educação física deve pensar nesses estágios em um continuum de tempo de prática, pois as mudanças nos estágios são graduais e não ocorrem desvios abruptos entre eles (MAGILL, 2000).

Como na questão 9, perguntas relacionadas à classificação das habilidades motoras também foram facilmente respondidas. Segundo Magill (2000), essa abordagem de classificação consiste em categorizar as habilidades motoras pelo que elas têm em comum (MAGILL, 2000). Assim, a questão 11 foi construída segundo a dimensão do grupamento muscular envolvido, ou seja, tornando a divisão das habilidades motoras em grossa ou fina. A questão 12 foi elaborada de acordo com a diferença entre os movimentos em relação ao início e fim da ação, isto é, se a habilidade motora é classificada em discreta, seriada ou contínua. Por fim, a questão 13 , sobre as classes de habilidades motoras, foi pautada segundo a estabilidade do ambiente, dividindo as habilidades motoras em fechadas ou abertas. O conhecimento acerca das classificações das habilidades parece ter um papel importante na qualidade da atuação do professor de educação física, tendo em vista que a forma como o planejamento e a execução das habilidades motoras ocorre está estreitamente relacionada às características da habilidade.

Os participantes também tiveram uma alta frequência de acertos na maioria das questões que se referiam aos fatores, como na questão 16 em que $63,12 \%$ respondeu corretamente que a demonstração "permite a formulação de uma representação mental da ação a ser realizada". Segundo Tani et al. (2011), a demonstração possibilita ao aprendiz a obtenção de informações sobre a natureza da tarefa a ser realizada, focando na informação sobre o "como 
O conhecimento do professor de educação física sobre aprendizagem motora

Nathálya Gardênia de Holanda Marinho Nogueira • Diego Augusto Paulon • Bárbara de Paula

Ferreira • Lidiane Aparecida Fernandes • Guilherme Menezes Lage

fazer" (TANI et al., 2011). Assim, é um fator importante na prática do professor de educação física, independentemente do contexto (por exemplo, escola e outros ambientes). Em relação à questão 19 , sobre instrução verbal, $60,28 \%$ dos participantes responderam corretamente que "utilizar frases curtas e concisas a respeito da meta da habilidade, ou seja, pistas verbais" é a melhor maneira de fornecer instrução verbal a um aprendiz. Assim, o professor de educação física deve moderar a quantidade de informações fornecidas para não afetar o entendimento do aprendiz a respeito do objetivo da habilidade e focar em informações que chamem a atenção do aprendiz para o que é relevante sobre a habilidade (MAGILL, 2000).

Dentro das questões sobre o tipo de prática, na questão 17 os participantes deveriam responder se a prática mental era superior a qual ou quais condições de prática, 83,69\% dos participantes responderam que a prática mental era superior à ausência de prática. Prática mental se refere ao ensaio simbólico de uma prática física na ausência de movimentos musculares brutos (RICHARDSON, 1967). Segundo a Teoria da Aprendizagem Simbólica, o processo de imaginação pode funcionar como um sistema codificador para ajudar os aprendizes a entenderem e adquirirem melhores padrões de movimento (FONSECA et al., 2008). Em relação à questão 18 , sobre as práticas pelo todo ou em partes, $54,61 \%$ dos participantes responderam corretamente que para tarefas com alta complexidade e baixa organização é mais apropriado utilizar o método de prática em partes. Avaliar o grau de complexidade e de organização de uma habilidade e determinar como essas duas características se relacionam, ajuda o professor de educação física a decidir se deve utilizar a prática como um todo ou em partes. Segundo Magill (2000), se a habilidade for de baixa complexidade e de alta organização, a prática da habilidade como um todo será a melhor escolha. Em contrapartida, a prática em partes seria mais adequada para indivíduos que aprendem habilidades mais complexas com baixa organização (MAGILL, 2000). Assim, cabe ao professor de educação física utilizar um método mais eficiente de acordo com a habilidade a ser ensinada. 
O conhecimento do professor de educação física sobre aprendizagem motora Nathálya Gardênia de Holanda Marinho Nogueira • Diego Augusto Paulon • Bárbara de Paula Ferreira • Lidiane Aparecida Fernandes • Guilherme Menezes Lage

Algumas questões tiveram alta frequência de respostas incorretas, entre elas estava a questão 7 , relacionada aos conceitos sobre a área do Comportamento Motor. Cerca de 42,55\% dos participantes responderam que "todas as alternativas anteriores" seriam conceitos que se referiam à Aprendizagem Motora, que procura estudar processos e mecanismos relacionados à aquisição de habilidades motoras e os fatores que a influenciam (TANI, 1998). Na questão 8, era permitido escolher duas alternativas em relação às teorias que explicam a aprendizagem motora, mas as duas opções destacadas foram a Teoria de Esquema de Schmidt e o Modelo de Ampulheta de Gallahue e Osmuz, 33,33\% e 34,75\% das respostas, respectivamente. No entanto, em vez do modelo de Ampulheta deveria ser a Teoria do Circuito Fechado de Adams, que alcançou apenas $18,44 \%$ das respostas. O modelo de Ampulheta está relacionado a outra área do Comportamento Motor, denominada Desenvolvimento Motor, que é definido como mudanças que ocorrem no movimento do ser humano ao longo do seu ciclo de vida (TANI, 1998). Apesar dos campos específicos de estudo Aprendizagem Motora, Controle Motor e Desenvolvimento Motor poderem ser caracterizados, é muito difícil separá-los em termos de fenômeno, pois estão intimamente relacionados (TANI, 1998). Dessa forma, é fundamental que o professor de educação física tenha uma visão integrada desses três fenômenos, além do conhecimento sobre suas possíveis linhas de investigação, modelos e teorias.

A questão 10 também teve uma alta frequência de respostas incorretas, pois sobre os fatores que o professor pode manipular para favorecer a aprendizagem motora, a resposta correta era "prática mental, feedback extrínseco, demonstração, organização da prática", que obteve apenas $19,15 \%$ das respostas. A alternativa com maior percentual de respostas para essa questão foi "feedback extrínseco, observação, organização da prática, instrução verbal", com 37,59\% das respostas, mas a observação não é um fator manipulável. Outra questão com alto índice de respostas incorretas foi a 14, ela estava relacionada ao feedback, mais es- 
O conhecimento do professor de educação física sobre aprendizagem motora Nathálya Gardênia de Holanda Marinho Nogueira • Diego Augusto Paulon • Bárbara de Paula Ferreira • Lidiane Aparecida Fernandes • Guilherme Menezes Lage

pecificamente sobre a melhor maneira de fornecê-lo em relação às características do movimento responsáveis pelo resultado do desempenho, que seria o CP. Segundo Corrêa et al. (2005), o CP é a informação sobre as características do movimento, as quais têm forte relação com o desempenho. No entanto, essa alternativa recebeu apenas $17,73 \%$ das respostas. Além disso, $49,65 \%$ dos participantes afirmaram que "todas as opções acima" (isto é, $C R$, $\mathrm{CP}$ e demonstração) eram as maneiras de informar sobre as características do movimento realizado. No entanto, feedback significa qualquer tipo de informação sensorial sobre o movimento, não exatamente com referência a erros. São informações que estão disponíveis como resultados do movimento e que são repassadas ao aprendiz (CORRÊA et al., 2005). Esse movimento executado é observado e avaliado pelo professor de educação física, que utiliza as informações obtidas para auxiliar o aprendiz nas próximas tentativas. Assim, se estabelece a dinâmica de ensino-aprendizagem (TANI, 1989). Dessa forma, feedback é a informação fornecida após o movimento, diferentemente das informações prévias à realização da ação, como a demonstração.

Por fim, apenas uma questão não gerou um padrão nas respostas, pois, na questão 15, sobre qual a melhor forma de estruturar a prática de uma ou mais habilidades motoras dentro da aprendizagem motora, $41,84 \%$ dos participantes afirmaram ser por meio da prática variada aleatória e $49,65 \%$ responderam ser por meio da prática constante. No entanto, na literatura sobre estruturação da prática, os estudos mostram que a prática variada leva a uma aprendizagem superior em relação à prática constante (APOLINÁRIO-SOUZA et al., 2019), exige maior esforço cognitivo (BICALHO et al., 2019; LELIS-TORRES et al., 2017) e tem sido mais associada a receptores glutamatérgicos (por exemplo, NMDA e AMPA) envolvidos em alterações no córtex motor que ocorrem ao longo do processo de aprendizagem motora (APOLINÁRIO-SOUZA et al., 2019). Assim, a forma pela qual a prática é estruturada fornece níveis distintos de aprendizagem e interfere na qualidade e quantidade de informações que são recebidas, processadas e 
O conhecimento do professor de educação física sobre aprendizagem motora Nathálya Gardênia de Holanda Marinho Nogueira • Diego Augusto Paulon • Bárbara de Paula Ferreira • Lidiane Aparecida Fernandes • Guilherme Menezes Lage

gerenciadas pelo aprendiz (GONÇALVES et al., 2007). Sendo a estruturação de prática um dos fatores mais importantes na aprendizagem motora (LAGE et al., 2011) era esperado uma maior frequência de acertos.

Concluindo o questionário, $86,52 \%$ dos participantes responderam que a aprendizagem motora é muito importante para a intervenção em educação física. Entender os processos subjacentes às mudanças no comportamento motor que resultam da prática, além dos fatores que a influenciam (TANI, 1998; TANI et al., 2010), é fundamental para o professor de educação física. Dessa forma, se pode promover atividades motoras em diversos contextos, optar por certas atividades e ações motoras em grupos determinados visando a melhor qualidade no ensino de habilidades motoras.

\section{Conclusão}

Este estudo investigou o nível de conhecimento dos graduados e graduandos em Educação Física sobre a área da Aprendizagem Motora. A maioria dos participantes afirmou que sabia o que era Aprendizagem Motora. No entanto, apresentaram dificuldades em relação a algumas informações sobre a parte teórica, conceito, teorias e fases da aprendizagem. Além disso, os participantes apresentaram certa dificuldade também em responder questões relacionadas aos fatores que influenciam a aprendizagem motora, principalmente os fatores que o professor manipula a respeito do feedback e da estruturação da prática. Apesar do conhecimento sobre a área da Aprendizagem Motora, principalmente em relação aos fatores, ser de extrema importância e contribuir efetivamente na forma segundo a qual o professor de educação física planeja e conduz as suas aulas e possíveis intervenções, a amostra do presente estudo apresentou dificuldade em responder às questões sobre a temática. Ademais, em relação às respostas corretas, não se sabe se os participantes o fizeram de forma intuitiva ou por realmente deterem esse conhecimento. 
O conhecimento do professor de educação física sobre aprendizagem motora Nathálya Gardênia de Holanda Marinho Nogueira • Diego Augusto Paulon • Bárbara de Paula Ferreira • Lidiane Aparecida Fernandes • Guilherme Menezes Lage

\section{Referências}

APOLINÁRIO-SOUZA, T. et al. Molecular Mechanisms Associated with the Benefits of Variable Practice in Motor Learning. Journal of Motor Behavior, p. 1-12, 2019. doi:10.1080/00222895.2019.16 49997.

ARAUJO, M. P. et al. Contribuição de diferentes conteúdos das aulas de Educação Física no ensino fundamental I para o desenvolvimento das habilidades motoras fundamentais. Revista Brasileira de Medicina do Esporte, São Paulo, vol. 18, n. 3, p. 153-157, 2012. doi:10.1590/S1517-86922012000300002.

BICALHO, L. E. A. et al. Oculomotor behavior and the level of repetition in motor practice: Effects on pupil dilation, eyeblinks and visual scanning. Human Movement Science, vol. 64, p. 142-152, 2019. doi:10.1016/j.humov.2019.02.001.

CORRÊA, U. C. et al. Efeitos da frequência de conhecimento de performance na aprendizagem de habilidades motoras. Revista Brasileira de Educação Física e Esporte, São Paulo, vol. 19, n. 2, p. 127-141, 2005. doi:10.1590/S1807-55092005000200004.

FITTS, P. M.; POSNER, M. I. Human performance. Belmont: Brooks/Colemann, 1967.

FONSECA, F. S. et al. Demonstração e prática mental na aquisição de habilidades motoras. Motricidade, vol. 4, n. 2, p. 61-66, 2008. doi:10.6063/motricidade.4(2).512.

GONÇALVES, W. R. et al. O efeito da interferência contextual em idosos. Revista Portuguesa de Ciências do Desporto, vol. 7, n. 2, p. 217-224, 2007.

LAGE, G. M. et al. O efeito da interferência contextual na aprendizagem motora: contribuições científicas após três décadas da publicação do primeiro artigo. Revista Brasileira de Ciência e Movimento, vol. 19, n. 2, p. 107 - 119, 2011. doi:10.18511/rbcm. v19i2.1711. 
O conhecimento do professor de educação física sobre aprendizagem motora Nathálya Gardênia de Holanda Marinho Nogueira • Diego Augusto Paulon • Bárbara de Paula Ferreira • Lidiane Aparecida Fernandes • Guilherme Menezes Lage

LELIS-TORRES, N. et al. Task engagement and mental workload involved in variation and repetition of a motor skill. Scientific reports, vol. 7, n. 1, p. 147-64, 2017. doi:10.1038/s41598-01715343-3.

LIN, C. H. J. et al. Interleaved practice enhances skill learning and the functional connectivity of fronto-parietal networks. Human Brain Mapping, vol. 34, n. 7, p. 1542-58, 2013. doi:10.1002/ hbm.22009.

MAGILL, R. A. Aprendizagem Motora: conceitos e aplicações. 5rd ed. São Paulo: Editora Edgard Blücher Ltda, 2000, p. 369.

PÚBLIO, N. S.; TANI, G.; MANOEL, E. J. Efeitos da demonstração e instrução verbal na aprendizagem de habilidades motoras na ginástica olímpica. Revista Paulista de Educação Física, vol. 9, n. 2, p. I11-I24, 1995. doi:10.11606/issn.2594-5904.rpef.1995.139474. RICHARDSON, A. Mental Practice: A Review and Discussion Part I. Research Quarterly. American Association for Health, Physical Education and Recreation, vol. 38, n. 1, p. 95-107, 1967. doi:10.1 080/10671188.1967.10614808.

SANTOS-NAVES, S. P. et al. Efeito da demonstração distribuída na aprendizagem do saque do voleibol. Revista Brasileira de Educação Física e Esporte, vol. 28, n. 4, p. 629-639, 2014. doi:10.1590/1807-55092014000400629.

TANI, G. Aprendizagem motora: tendências, perspectivas e problemas de investigação. Revista galego-portuguesa de psicoloxía e educación, vol. 2, n. 2, p. 199-215, 1998.

TANI, G. Significado, detecção e correção do erro de performance no processo ensino-aprendizagem de habilidades motoras. Revista Brasileira de Ciência e Movimento, vol. 3, n. 1, p. 50-8, 1989. doi: 10.18511/rbcm.v3i4.112.

TANI, G. et al. O estudo da demonstração em aprendizagem motora: estado da arte, desafios e perspectivas. Revista Brasileira Cineantropometria e Desempenho Humano, Florianópolis, vol. 13, n. 5, p. 392-403, 2011. doi:10.5007/1980-0037.2011v13n5p392. 
O conhecimento do professor de educação física sobre aprendizagem motora

Nathálya Gardênia de Holanda Marinho Nogueira • Diego Augusto Paulon • Bárbara de Paula

Ferreira • Lidiane Aparecida Fernandes • Guilherme Menezes Lage

TANI, G.; JUNIOR, C. M. M.; GOMES, F. R. F. Frequência, precisão e localização temporal de conhecimento de resultados e o processo adaptativo na aquisição de uma habilidade motora de controle da força manual. Revista Portuguesa de Ciências do Desporto, vol. 5, n. 1, p. 59-68, 2005. doi:10.5628/rpcd.05.01.59.

TANI, G. et al. Pesquisa na área de comportamento motor: modelos teóricos, métodos de investigação, instrumentos de análise, desafios, tendências e perspectivas. Revista da Educação Física/ UEM, Maringá, vol. 21, n . 3, p. 1-51, 2010. doi:10.4025/reveducfis. v21i3.9254.

TONELLO, M. G. M.; PELLEGRINI, A. M. A utilização da demonstração para a aprendizagem de habilidades motoras em aulas de educação física. Revista Paulista de Educação Física, vol. 12, n. 2, p. 107-114, 1998. doi:10.11606/issn.2594-5904. rpef.1998.139538.

UGRINOWITSCH, H.; BENDA, R. N. Contribuições da Aprendizagem Motora: a prática na intervenção em Educação Física. Revista Brasileira de Educação Física e Esporte, São Paulo, vol. 25, n. especial, p. 25-35, 2011. doi:10.1590/S1807-55092011000500004.

\section{Aprovação de comitê de ética em pesquisa}

Pesquisa aprovada pelo Comitê de Ética da Universidade Federal de Viçosa. Título: CONHECIMENTO DOS PROFESSORES E ALUNOS DE EDUCAÇÃO FÍSICA SOBRE ASPECTOS RELACIONADOS AO COMPORTAMENTO MOTOR.

\section{Publisher}

Universidade Federal de Goiás. Faculdade de Educação Física e Dança. Publicação no Portal de Periódicos UFG. As ideias expressadas neste artigo são de responsabilidade de seus autores, não representando, necessariamente, a opinião dos editores ou da universidade. 